\title{
Proyecto de investigación INVISO: industrialización de viviendas sostenibles
}

\author{
INVISO research project: industrialization of \\ sustainable houses
}

L. Queipo $^{(*)}$, J. M. Navarro ${ }^{(* *)}$, M. Izquierdo ${ }^{(*)}$, A. del Águila ${ }^{(* *)}$, D. Guinea ${ }^{(* * *)}$, M. Villamor ${ }^{(* * * *)}$, S. Vega ${ }^{(* *)}$, J. Neila $a^{(* *)}$

RESUMEN

El proyecto singular y estratégico INVISO (Industrialización de viviendas sostenibles), que comenzó en el año 2006, pretende optimizar la producción de viviendas por medio de la industrialización de los procesos constructivos, generando el diseño de nuevos materiales y sistemas y elaborando herramientas de diseño y gestión que faciliten este proceso.

195-13

Palabras clave: industrialización, sostenibilidad, concurso de ideas, vivienda.

\section{INTRODUCCIÓN}

El proyecto de investigación INVISO (Industrialización de Viviendas Sostenibles) es un Proyecto Singular y Estratégico financiado por el Ministerio de Ciencia e Innovación. Comenzó en el año 2006 liderado en un principio por el Instituto de Ciencias de la Construcción Eduardo Torroja y actualmente por la empresa DRAGADOS. El carácter Singular del proyecto se encuentra, no sólo en sus objetivos, como se indica a continuación, sino en la multidisciplinariedad de los socios participantes y la consiguiente importancia de potenciar y posibilitar una sólida y fluida coordinación del trabajo de Investigación desarrollado por los diferentes actores involucrados: empresas, OPIs y asociaciones etc... por una parte, $y$, dentro de ellos, por los diferentes profesionales involucrados, desde el Arquitecto proyectista hasta los constructores, pasando por los fabricantes

\section{SUMMARY}

The singular and strategic INVISO (Spanish acronym for "industrialization of sustainable housing") project begun in 2006 aims to optimize housing production by industrializing construction processes, furthering the design of new materials and systems and formulating design and management tools to facilitate this process.

Keywords: industrialization, sustainability, ideas competition, housing.

de materiales, diversos suministradores, etc... y llegando especialmente al factor más importante en la cadena de valor que es el usuario final de la vivienda, ya que es éste hacia quien, en definitiva, van dirigidos los desarrollos de este proyecto. No debe olvidarse que es el usuario final, el ciudadano, quien va a disfrutar del producto y debe, por tanto, ver el resultado como algo apetecible y que le aporte ventajas. El Objetivo general del proyecto no ha variado a lo largo del desarrollo del mismo y presenta dos vertientes fundamentales:

a. Asegurar la producción más eficiente y competitiva de viviendas por medio de la industrialización de los procesos constructivos, generando el diseño de nuevos materiales y sistemas, y desarrollando una serie de herramientas de diseño y gestión que faciliten la labor, tanto a los promotores y proyectistas, como a los fabricantes y constructores.

\footnotetext{
(*) Instituto de Ciencias de la Construcción Eduardo Torroja (CSIC) (Madrid, España)

${ }^{(*)}$ Dragados, S. A. (Madrid, España)

${ }^{(* * *)}$ Dpto. de "Construcción y Tecnología Arquitectónicas" de la Escuela T. S. de Arquitectura. U. P. M. (Madrid, España)

${ }^{(* * * *)}$ Instituto de Automática Industrial (CSIC) (Madrid, España)

${ }^{(* * * *)}$ Nemetschek (España)
} 
b. Asegurar la sostenibilidad de las viviendas, tanto en su proceso de producción como en su uso, desarrollando el diseño de elementos que pueda ofrecer el mercado para incorporar en la construcción de las mismas, que aseguren su funcionamiento eficiente, así como los sistemas de control domótico que posibiliten al usuario la funcionalidad óptima de las viviendas y su mantenimiento.

c. Abordar el desarrollo y diseño de soluciones constructivas modulares reales con un máximo aprovechamiento energético y extremadamente respetuosas con el medio ambiente.

Este triple objetivo ha sido ya perseguido en múltiples ocasiones anteriores a INVISO sin éxito. Probablemente las razones de estos fracasos anteriores se fundamentan en que históricamente se ha tratado de abordar este objetivo de implantar los sistemas industrializados en el sector de la construcción tradicional de forma parcial, sin tener en cuenta el conjunto total de intervinientes en el proceso, desde el usuario hasta el mantenedor, pasando por el proyectista, el constructor o los suministradores de material. Estos actores desempeñan cada uno un rol específico y que debe ser tenido en cuenta a la hora de intentar modificar una parte o la totalidad del proceso constructivo.

Este planteamiento es una de las razones que hacen a este un proyecto Estratégico. Es más, tan sólo desde ese planteamiento se pueden alcanzar los objetivos perseguidos.
En el Cuadro 1 se muestra la distribución de subproyectos que componen el proyecto INVISO en el 2008 incluyendo todos los socios participantes en cada subproyecto y sus respectivos líderes.

Partiendo de los objetivos generales del proyecto y siempre teniendo presente la necesidad de mejorar el nivel tecnológico y la productividad en el sector de la construcción, así como aumentar el nivel de eficiencia energética y el confort para el usuario de la vivienda en este país, el PROYECTO INVISO tiene como objetivos específicos los siguientes:

- Desarrollo de nuevos procesos constructivos y optimización de los existentes mediante la automatización de éstos, con las consiguientes mejoras en precisión de ejecución, niveles de productividad, especialización de mano de obra, etc., promoviendo la industrialización del proceso global.

- Definición y desarrollo de nuevas soluciones técnicas sostenibles que puedan ser incorporadas en las viviendas mejorando su eficiencia de uso, abaratando su mantenimiento, reduciendo el impacto ambiental, etc.

- Definición de unos diseños tipológicos basados en criterios de alta industrialización y sostenibilidad, maximizando la flexibilidad y el confort y presentando a su vez dimensiones basadas en un claro convenio modular que permita altos niveles de estandarización en cuanto a los elementos constructivos que incorporen.

CUADRO 1

\begin{tabular}{|c|c|c|c|c|c|c|c|c|c|c|c|c|c|c|c|c|c|c|c|c|}
\hline \multirow{3}{*}{$\begin{array}{l}\text { PROPUESTA } 2008 \\
\text { INVISO }\end{array}$} & \multicolumn{5}{|c|}{ OPIS } & \multirow{2}{*}{\multicolumn{2}{|c|}{$\begin{array}{c}\text { EMPRESAS } \\
\text { MUNICIPALES }\end{array}$}} & \multirow{2}{*}{\multicolumn{3}{|c|}{$\begin{array}{c}\text { CENTROS } \\
\text { TECNOLOGICOS Y } \\
\text { ASOCIACIONES S. ALL } \\
\end{array}$}} & \multicolumn{10}{|c|}{ EMPRESAS PRIVADAS } \\
\hline & \multicolumn{3}{|c|}{ UPM } & \multicolumn{2}{|c|}{ CSIC } & & & & & & \multicolumn{4}{|c|}{ GRAN EMPRESA } & \multicolumn{3}{|c|}{ MEDIANA EMPRESA } & \multicolumn{3}{|c|}{ PEQUEÑA EMPRESA } \\
\hline & TISE & EISAM & ABBO & EETCC & IAI & EWNS & GICAMAN & ANDECE & ITeC & CIDEMCO & DRAGADOS & SEIS & IDOM & ORTIZ & INTEMPER & $\begin{array}{c}\text { NEMETSC } \\
\text { HEK } \\
\end{array}$ & VEKA & $\begin{array}{l}\text { BLUE } \\
\text { COAST } \\
\end{array}$ & GOP & CAMAC \\
\hline $\begin{array}{l}\text { Subproyecto } 1 \\
\text { ANALLISIS DEL ENTORNO SO- } \\
\text { CIOECONÓMICO } \\
\text { COO RDINACIÓN } \\
\text { Y DIFUSIÓN }\end{array}$ & & & & & & & & & & & & & & & & & & & & \\
\hline Subproyecto 2 & & & & & & & & & & & & & & & & & & & & \\
\hline $\begin{array}{l}\text { Subproyecto } 3 \\
\text { GENERACIÓN SOSTENIBLE DI } \\
\text { ENERGIIA EN VIVIENDAS }\end{array}$ & & & & & & & & & & & & & & & & & & & & \\
\hline $\begin{array}{l}\text { Subproyecto } 5 \\
\text { OPIIMIZACIÓN DE LA INDUS } \\
\text { TRIALIZACIÓN EN LA CONNS- } \\
\text { TRUCCIÓN DE VIVIENDAS } \\
\end{array}$ & & & & & & & & & & & & & & & & & & & & \\
\hline $\begin{array}{l}\text { Subproyecto } 6 \\
\text { AUTOMAIIZACIÓN } \\
\text { DE LA CONSTRUCCIÓN }\end{array}$ & & & & & & & & & & & & & & & & & & & & \\
\hline $\begin{array}{l}\text { Subproyecto } 7 \\
\text { DESARROLLO DE } \\
\text { HERRAMIENTAS } \\
\text { INFORMÁTICAS }\end{array}$ & & & & & & & & & & & & & & & & & & & & \\
\hline $\begin{array}{l}\text { Suboproyecto } 9 \\
\text { DISENOY EXPERIMENTACIÓN } \\
\text { DESOLUCIONES TÉCNICAS } \\
\text { INNOVADORAS } \\
\end{array}$ & & & & & & & & & & & & & & & & & & & & \\
\hline $\begin{array}{l}\text { Subproyecto } 10 \\
\text { SISTEMAS PARA LA } \\
\text { OPTIMIZACIÓN DEL } \\
\text { COMPORTAMIENTO } \\
\text { EFICIENTE DE LAS VIVIENDAS }\end{array}$ & & & & & & & & & & & & & & & & & & & & \\
\hline
\end{tabular}


- Desarrollo de una herramienta informática que incorpore todos los desarrollos anteriormente expuestos y permita desde el principio del proceso global constructivo, a partir de los diseños tipológicos definidos en el proyecto e incluyendo las soluciones técnicas óptimas presentar una propuesta sostenible e industrializada para cualquier proyecto constructivo de viviendas.

Recordando que el objetivo del proyecto se basa en:

- Optimizar la producción y mantenimiento de viviendas

- Alcanzar la máxima sostenibilidad en la producción y uso de viviendas

El proyecto INVISO fue estructurado en su momento, y lo sigue estando, de manera que los distintos subproyectos se agrupan en un primer nivel en dos conjuntos mayores. Cada uno de estos conjuntos da mayor importancia a uno de los dos objetivos y la integración de los SP que forman cada conjunto es esencial para el logro de dicho objetivo.

A un segundo nivel, dado el carácter estratégico del proyecto, es básico el asegurar el continuo flujo de información entre ambos conjuntos e integrar sus resultados.

La vertiente "Industrializadora" del proyecto se encuentra representada por el conjunto de subproyectos:

- SP2 DISEÑO DE TIPOLOGÍAS DE SOLUCIONES RACIONALIZADAS

- SP5 OPTIMIZACIÓN DE LA INDUSTRIALIZACIÓN EN LA CONSTRUCCIÓN DE VIVIENDAS

- SP6 AUTOMATIZACIÓN DE LA CONSTRUCCIÓN

- SP7 DESARROLLO DE hERRAMIENTAS INFORMÁTICAS

Las funciones específicas de estos cuatro subproyectos se han integrado con el fin de Optimizar el proceso global de producción y mantenimiento de viviendas desarrollando tareas específicas que afecten a todas las fases del buscado proceso de producción, obsérvese que se habla de proceso de producción de viviendas y no de construcción de viviendas, potenciando el aumento del grado de industrialización y automatización del mismo.

La segunda vertiente del INVISO se centra en la "sostenibilidad" de las viviendas producidas. Sostenibilidad tratada desde diferentes ángulos, ahondando en temas de eficiencia energética, reducción de impacto medioambiental, flexibilización de la vivienda para adaptarla a los cambios sociales, proponiendo soluciones bioclimáticas que promuevan el ahorro energético, fomentando el uso de energías renovables, etc.

Este conjunto lo componen los siguientes subproyectos:

- SP3 Generación SOSTENIBLE DE ENERGÍA EN VIVIENDAS

- SP9 DISEÑO Y EXPERIMENTACIÓN DE SOLUCIONES TÉCNICAS INNOVADORAS

- SP10 SISTEMAS PARA LA OPTIMIZACIÓN DEL COMPORTAMIENTO EFICIENTE DE LAS VIVIENDAS

Parece evidente que ambas líneas, a pesar de ser sustancialmente diferentes, se complementan a la hora de cumplir el objetivo global que se persigue a través de este proyecto que es el de mejorar el sector de la construcción de manera conjunta, ahondando no sólo en la parte del proceso que precede a la entrega de una vivienda sino cubriendo su ciclo de vida asegurando unos niveles de operación y rendimiento óptimos de las mismas.

Como complemento a estos dos conjuntos de subproyectos se encuentra el SUBPROYECTO 1 ANÁLISIS DEL ENTORNO SOCIOECONÓMICO, COORDINACIÓN Y DIFUSIÓN, encargado de la difusión de resultados y logros del proyecto a través de artículos, ponencias, charlas, jornadas técnicas, etc., $y$, por otro lado, de la coordinación del proyecto en su totalidad, asegurando la interacción e integración de los subproyectos entre sí, de manera que los avances generados puedan ser difundidos de manera conjunta como desarrollos INVISO.

En el marco del subproyecto SP1, se avanza en el objetivo de acotar las bases técnicas fundamentales sobre las que cimentar un posible "Sello INVISO" que tiende a valorar o diferenciar en forma fácilmente asimilable la "optimización de la producción de viviendas". Bases que, de forma justificada e inequívoca, permiten cuantificar el nivel o grado de cumplimiento desde una doble óptica, la "sostenibilidad"y/o "industrialización" en el sentido más amplio, tanto de proyectos de viviendas como de realizaciones ejecutadas. El estado del arte en este objetivo concreto a nivel internacional, muestra mucha más actividad en lo que se refiere a la "sostenibilidad" que a la "industrialización", prácticamente inactivo. Existe además un SP8 EJECUCIÓN DE EDIFICIOS DE DEMOSTRACIÓN que no se propone comenzar en este periodo debido a la necesidad de maduración de algunos de los resultados logrados hasta ahora en el proyecto. La demostración física de alguna de 
las estrategias bioclimáticas investigadas, de varias de las soluciones técnicas desarrolladas así como de alguna de las propuestas tipológicas definidas entre el 2007 y 2008 se antoja vital para la validación general de la viabilidad del proyecto y por ello es un SP que se irá estructurando este año y sería incluido en una potencial propuesta para el 2009.

\section{DISEÑO DE TIPOLOGÍAS DE SOLUCIONES RACIONALIZADAS DE VIVIENDAS (Subproyecto 2)}

Los avances en la industrialización de la construcción de viviendas deben apoyarse en todos aquellos aspectos que influyen en su proceso. Entre ellos, el proyecto constituye el eje que puede hacer factible, o no, la industrialización. El proyecto debe definir no sólo la geometría y funcionalidad del edificio, sino también su solución constructiva; lo que implica que si no se plantea su construcción mediante un proceso industrializado desde su concepción, resultará muy difícil la adaptación posterior para su ejecución por métodos industrializados.

Por tanto, en aras de la facilitación de aplicación de procesos industrializados en la producción de viviendas mediante el estudio y adaptación de los proyectos en su etapa de diseño, se ha trabajado en la concepción de propuestas tipológicas de viviendas con unas determinadas características que les permitan por un lado, cubrir de la manera más eficiente posible las necesidades y requerimientos de los usuarios de las mismas a lo largo de periodos de tiempo en los que dichas necesidades pueden cambiar radicalmente; y, por otro lado, de permitir la ejecución constructiva de las mismas mediante el uso de elementos y sistemas constructivos

1. Distintas configuraciones de unidades de vivienda. prefabricados del mercado nacional.
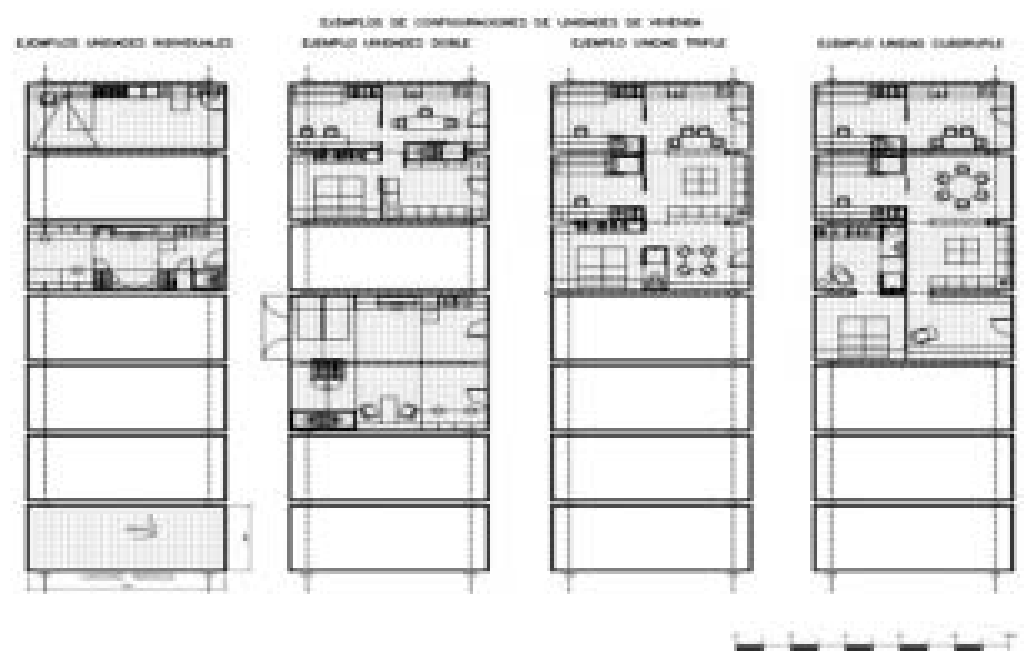

Los conceptos estudiados para el desarrollo de dichas propuestas son parte del continuo debate entre los arquitectos proyectistas y los agentes participantes en el proceso dado que no los consideran resueltos en las viviendas proyectadas y construidas en la actualidad: flexibilidad de diseños, modularización de dimensiones, versatilidad de aplicaciones, viviendas y fachadas perfectibles, alternativas a la duplicación completa de los núcleos sanitarios, luces y disposiciones estructurales, compartimentaciones ambiguas, tabiquerías y divisiones móviles, participación del usuario, planteamientos bioclimáticos, etc.

Todo este estudio ha posibilitado el diseño de un abanico de soluciones compatibles entre sí que facilitarán la construcción de viviendas industrializadas teniendo como meta final la cadena de montaje e incrementará, en cada paso, su sostenibilidad. Las características principales que reúnen las propuestas desarrolladas son, entre otras:

- Regularización de la estructura dentro de una trama modular, con miras a la estandarización del tamaño y forma de los elementos, así como a su posible intercambio, sustitución o reciclaje, sin impedir la adecuada organización de sótanos o garajes.

- Análisis de las asignaciones funcionales de las piezas de la vivienda, con objeto de optimizar la superficie final sin impedir las transformaciones que inevitablemente ha de sufrir cualquier vivienda a lo largo de su vida.

- Estandarización de los paneles de fachadas, particiones, mobiliario y paneles interiores con vistas a su prefabricación.

- Reconsideración de las instalaciones, dada su creciente complejidad y variedad mediante:

- Rediseño de los "locales húmedos" e instalaciones de acometidas y desagües

- Reconsideración y optimización del/los sistema/as de ventilación con un objetivo similar

- Diseño de canalizaciones y regletas modulares registrables para alojar las instalaciones eléctricas, de telecomunicación y de los distintos líquidos (agua caliente y fría) y gases (combustión, refrigeración...), previa su agrupación y compatibilidad.

- Consideración de las posibilidades bioclimáticas del edificio, en función de su ubicación geográfica.

El siguiente paso en este estudio, que se desarrolla a lo largo del 2008, es la implementación de alguna de las propuesta desarrolladas en un proyecto real que pueda ser presentado como alternativa a un proyecto tradicional, validando así las ventajas que un diseño 
racionalizado junto con su consiguiente producción industrializada aporta al proceso de construcción de viviendas tal y como hay lo conocemos.

\section{GENERACIÓN SOSTENIBLE DE ENERGÍA EN VIVIENDAS (Subproyecto 3)}

El consumo energético de España en 2007 fue de unos $280.00010^{6} \mathrm{kWh}$ de electricidad; $290.000 \cdot 10^{6} \mathrm{kWh}$ de gas natural y $60 \cdot 10^{9} \mathrm{~kg}$ de productos petrolíferos. Este consumo generó una emisión de $385 \cdot 10^{6}$ toneladas de dióxido de carbono, 2,6\% menos que en 2005 que para una población de $45 \cdot 10^{6}$ habitantes, corresponde una emisión media anual por español de 8,6 toneladas de $\mathrm{CO}_{2}$. El Protocolo de Kioto permite que la emisión de $\mathrm{CO}_{2}$ en 2012 sea el 15\% superior a la emisión correspondiente al año 1990. La emisión española de $\mathrm{CO}_{2}$ en 1990 fue de $260 \cdot 10^{6}$ y en 2012 debería ser de $300 \cdot 10^{6}$ toneladas, aproximadamente. Según el IDAE, el consumo de electricidad en la climatización de edificios supone el $12 \%$ del total, aumentando el consumo en las viviendas que ya asciende al $2-3 \%$ y tiene tendencia creciente.

Muy recientemente, el Comité ITRE en el Parlamento Europeo prepara establecer para Europa, en el 2020, mínimos de energías renovables en los edificios para calor y frío. Además, el entorno de I+D en Europa sobre estos temas es muy favorable, véase Intelligent Energy Europe Programme. Por lo tanto, puede decirse que la propuesta de tratar de acometer medidas de ahorro y eficiencia energética, conjuntadas con experiencias y estudios de aplicación, separada o conjunta de energías renovables, contribuirá a mitigar la actual dependencia energética de españa (70\%), de Europa (50\%) así como a reducir las emisiones de GEI (Protocolo de Kioto).

Por otra parte, actualmente, en España, se está tramitando el Reglamento de Seguridad para Instalaciones Frigoríficas e Instrucciones Técnicas Complementarias que contempla la sustitución de los CFC por otros refrigerantes como el R422A, R422D y R427A. Refrigerantes que son HFCs, no contienen cloro y por esta razón no destruyen el ozono. Pero son potentes gases de efecto invernadero (en adelante GEI), con un PEID unas 2.500 veces superior al del dióxido de carbono, lo que supone un $25 \%$ menor que los refrigerantes R22, R134a, R407C y R410A utilizados actualmente. Sin embargo, no se conocen aún resultados prácticos de su rendimiento en instalaciones. En concreto el R422D, según la empresa productora DuPont de Nemours, se podrá utilizar en aplicaciones de aire acondicionado residencial y comercial, para sustituir
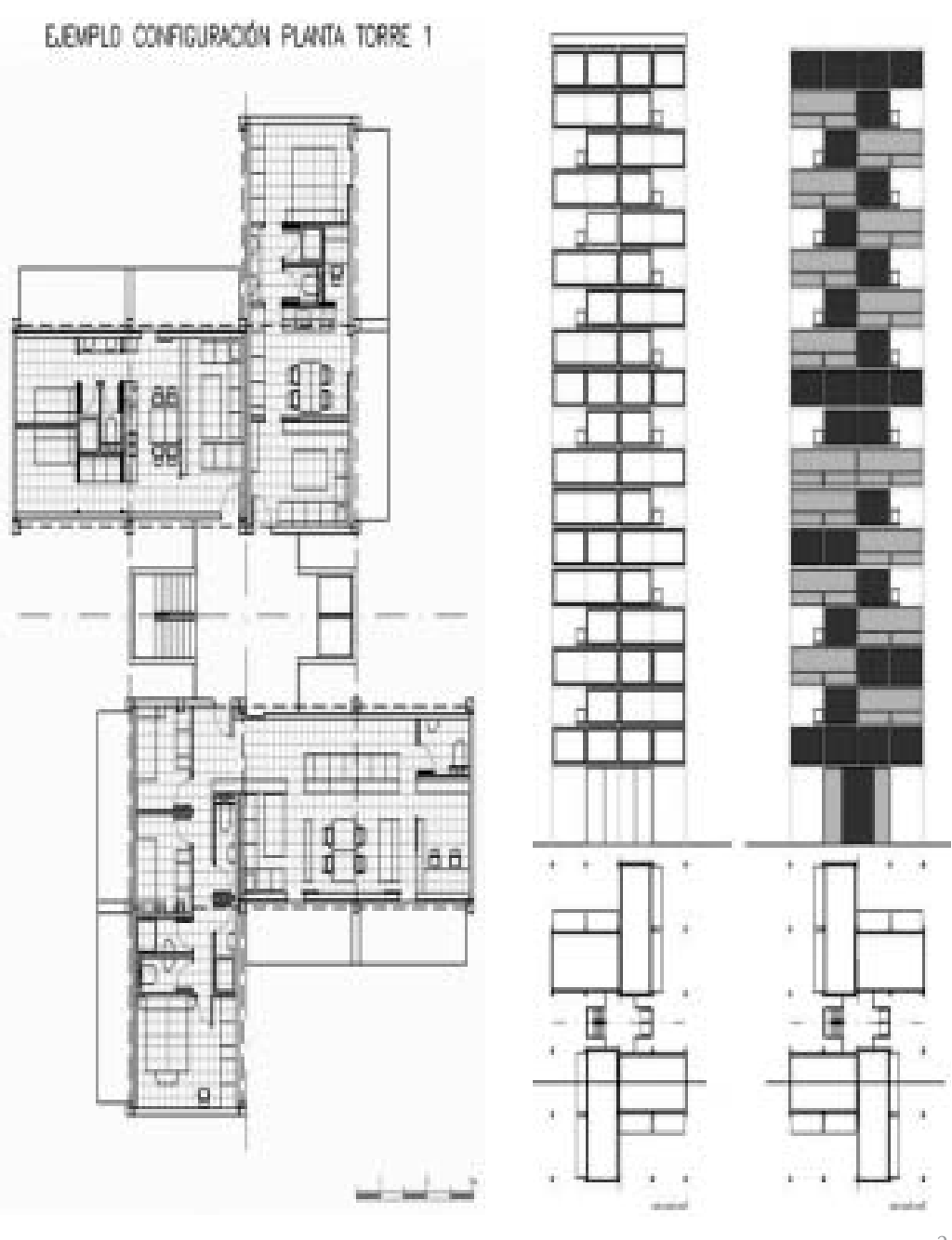

a los refrigerantes R22, R407C y R410A y sus ventajas son: no destruye el ozono, no se descompone en caso de fuga de la instalación, es compatible con los aceites lubricantes, trabaja con algo más de eficiencia que el R22 y su contribución como GEI es un $25 \%$ inferior a la de los actualmente utilizados. O sea que si los refrigerantes R22, R407C, R134a, R410A, actualmente en uso, son GEl con un potencial medio de unas 3.300 veces superior al del $\mathrm{CO}_{2}$, a horizonte temporal de 25 años, los nuevos refrigerantes que las autoridades de la UE nos proponen serán GEl "sólo" 2.500 veces superior a la del $\mathrm{CO}_{2}$ (Ref. 1, 2, 3, 4 y 5), página 8 . A los anteriores efectos hay que añadir el uso de agua para producir la electricidad, y el consumo de agua en las torres de enfriamiento. El uso de torres genera a su vez epidemias de legionellosis, enfermedad de aparición recurrente en los medios de comunicación. Podemos decir que existe una gran preocupación en las instituciones mundiales por los efectos contaminantes de la tecnología frigorífica y no parece fácil encontrar una solución aceptable.

No obstante, la sociedad actual no puede prescindir de la tecnología frigorífica, y por esta ra- 
zón es necesario crear sistemas de producción de frío que minimicen su impacto ambiental. Esto se puede conseguir utilizando la energía solar como fuente de calor para accionar las máquinas frigoríficas para la climatización de viviendas, y sustituir los refrigerantes fluorados, que denominaremos en general "refrigerantes orgánicos" por otro refrigerante, como el agua, que no crea estos problemas.

Los objetivos del subproyecto SP3 son: el desarrollo de instalaciones de energía solar para calefacción y refrigeración y la fabricación de máquinas frigoríficas de absorción que utilizan el agua como refrigerante, condensadas por aire, para evitar las epidemias de legionella, a la vez que son accionadas por energía solar, calores residuales o bio-combustibles.

\section{OPTIMIZACIÓN DE LA INDUSTRIALIZACIÓN EN LA CONSTRUCCIÓN DE VIVIENDAS (Subproyecto 5)}

En este subproyecto se persigue la optimización del proceso de producción de viviendas, mediante la aplicación de técnicas de industrialización.

Se trata de incorporar los procesos sistematizados de diseño con los sistemas industrializados de construcción de viviendas, basados en la industrialización abierta, esto es, utilizando componentes y sistemas industrializados procedentes de distintos fabricantes y que se integran en obra mediante procedimientos simples y rápido.

Para ello se considera imprescindible la puesta en marcha de dos convenios fundamentales:

1.-Adopción de un Convenio de coordinación dimensional y modular

2.-Adopción de Convenios de intercambiabilidad de componentes y de compatibilidad de juntas.

Además, y como parte fundamental del INVI$\mathrm{SO}$, se está procediendo a la creación de una Base de Datos a escala nacional, mediante la recopilación de todos los productos industrializados existentes en el mercado español.

Hay que llegar a una Edificación en que no sea necesario el empleo de agua en la ejecución de las unidades de obra en el "tajo", tendiéndolas a fabricarlas en factoría, sometiéndolas a un montaje rápido y sencillo. Hay que tender a hacer todas las fijaciones "en seco" (tornillería, soldadura, clips, etc.) y alcanzar que todas las conexiones en la construcción de interiores sean "en seco", sencillas y removibles en el tiempo.

El Objetivo principal al que están destinadas todas las tareas del SP \% es de aportar todos los datos y los criterios necesarios para llevar a buen puerto la finalidad del Proyecto INVISO DE alcanzar la optimizar el proceso de producción de viviendas de tipo industrializado, mediante innovación tecnológica y consideraciones de sostenibilidad a lo largo del ciclo de vida, consiguiendo al mismo tiempo la modernización del Sector de la Edificación, como se expica en la Introducción del Proyecto.

Con este fin se considera fundamental la creación de la Base de Datos, procediendo a la clasificación y catalogación de tipologías de sistemas, elementos y procedimientos constructivos industrializados.

Se está realizando una recopilación del más amplio espectro posible de elementos industrializados en el terreno de la construcción de edificios de viviendas, pudiendo efectuar una primera clasificación de los siguientes:

- "Elementos estructurales". entre éstos, a su vez, se debe realizar la división entre elementos lineales (fundamentalmente vigas y pilares), y elementos superficiales (forjados y escaleras)

- "Elementos de fachada". se realizará un catálogo de elementos de fachadas pesadas y ligeras, materiales de los mismos.

- "Elementos constructivos para la racionalización de las instalaciones": paneles y bloques técnicos y células sanitarias.

- "Sistemas de construcción de interiores" industrializados.

- "Elementos tridimensionales", con el objetivo de una terminación y un acabado integral en factoría, con labores mínimas y elementales de conexiones en obra.

Según los estudios realizados por el CSTB de París, se considera que la unidad de obra sobre la que se tiene que realizar un mayor esfuerzo de industrialización es la de obra gruesa, a la que se tiene que incorporar en fábrica, o facilitar su integración en obra, el mayor grado de acabados, incorporando, al menos, conductos y registros para instalaciones, así como las carpinterías.

La recopilación de los elementos anteriores se hace efectuando una priorización encaminada a establecer cuáles son los elementos más favorables para efectuar una industrialización global, a partir de una serie de criterios entre los que incluimos los siguientes:

- Modularidad

- Durabilidad 
- Eficacia estructural

- Eficacia funcional

- Estética y forma

- Aspectos medioambientales

- Materiales reciclados o reciclables, así como reutilizados y reutilizables

- Sostenibilidad

- Otros aspectos de tipo social

- Seguridad (en la construcción y en la utilización)

- Calidad de acabados

- Conectividad entre los distintos elementos

- Condiciones de uso

- Participación del usuario final

También se precisa el establecimiento de Convenios de Coordinación Dimensional y de Compatibilidad de juntas y conexiones, que posibilitan la integración en un edificio de componentes procedentes de distintos fabricantes.

A continuación se resaltan las tareas desarrolladas hasta el momento más destacables:

1.- Se comenzaron analizando la panorámica de los últimos años de la construcción industrializada en viviendas y de los convenios modulares existentes. Se ha procedido a hacer una radiografía de las investigaciones recientes en este ámbito y de la normativa vigente y en fase de desarrollo. La tarea también ha consistido en la creación de una base de datos y análisis de los sistemas, elementos e instalaciones industrializadas actualmente disponibles en el mercado.

2.- Se ha realizado un Informe sobre la industrialización de viviendas en los últimos quince años en España, Europa, EEUU y en Japón. Análisis de experiencias científicas europeas en la Industrialización Abierta en viviendas, ya que está íntimamente relacionadas con el proyecto INVISO. Como p.e. ManuBuild, ACC, Programa PIP, Programa SPRINT de la CEE. También se ha elaborado un Informe sobre las normativas vigentes en materia de industrialización de viviendas, en las normativas emergentes o en fase de redacción y un escenario futuro de la evolución de la normativa, y un Análisis inicial de los convenios modulares existentes (p.e. ACC, NEN).

3.- Se ha realizado en dicha tarea un análisis y una recopilación de sistemas industrializados más o menos cerrados, elementos constructivos abarcando toda la estructura y la envolvente del edificio, instalaciones industrializadas del tipo paneles técnicos, y células técnicas y todos los sistemas de construcción interiores. Se ha creado la Base de Datos inicial del proyecto.
Todo el material recopilado se ha sometido a un Análisis evaluador multicriterio para su posterior uso en la herramienta informática.

4.- Ha sido fundamental para la constitución de la base de datos la recogida de dichos datos en unas "fichas-tipo" para cada sistema o cada componente. Esto nos servirá de soporte para interrelacionar posteriormente en la Web campos vinculantes como pueden ser, datos de contacto, modelos de productos, experiencia construida desde el año 2000 y precios por $\mathrm{m}^{2}$ del panel.

\section{Se han realizado las "fichas-tipo" de la parte correspondiente a Sistemas constructivos industrializados que comprenden:}

1. Sistemas de paneles prefabricados de hormigón;

2. Sistemas de hormigonado in situ;

3. Sistemas de módulos tridimensionales.

Se han estudiado todos los sistemas que, en principio, ofrecían ciertas garantías de calidad, por prestigio en el mercado, posesión de documentos de calidad, habiéndose verificado con visitas a obras y fábricas, fundamental para fijar los criterios de evaluación.

De cada sistema se ha hecho una ficha de características, incluso gráficas, que valen, inicialmente, para su descripción y evaluación

5.- Se ha llevado a cabo el establecimiento de unos Convenios fundamentales de Coordinación modular y Dimensional.

Para ello ha valido la experiencia en este campo del Coordinador del Grupo TISE, que ha elaborado Normas AENOR sobre el tema.

Con todo ello se ha llevado a cabo la adopción de un Convenio de Coordinación dimensional y modular con una serie de criterios:

a) Tienen que ser simples y prácticos, para un uso eficaz.

b) Sólo para viviendas.

c) No pretender modular "dimensiones técnicas" (procedentes de cálculos y, por tanto, "amodulares").

d) Poner de acuerdo a fabricantes.

e) Poner de acuerdo a constructoresmontadores.

f) Suficientemente flexible para que permita unas composiciones estéticas y funcionales de viviendas.

Se ha analizado toda la normativa existente a escala nacional e internacional sobre 


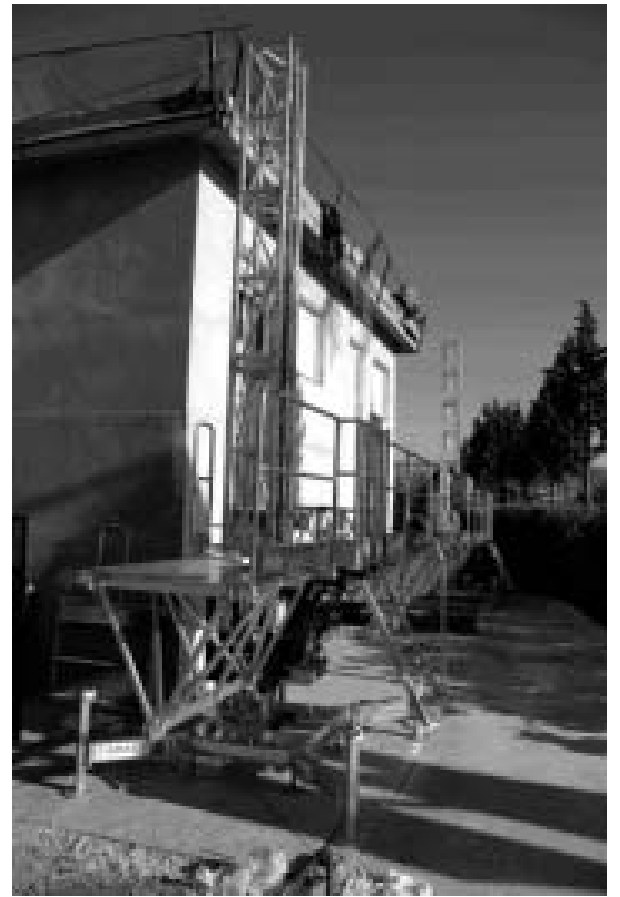

coordinaciones dimensional y modular llegando a unas propuestas muy sencillas, basadas principalmente en los Convenios franceses, modificados por la experiencia propia del Equipo.

Así se adopta:

1.- El MÓDULO-BASE $M=10$ cm. ó 100 mm, dependiendo de los materiales en que se esté usando, como unidad de crecimiento VERTICAL.

2.- El MULTIMÓDULO de $30 \mathrm{~cm}$, para la coordinación de las dimensiones HORIZONTALES. Éste es un multimódulo muy conocido y usado en arquitectura.

3.- El uso, únicamente, del SUBMÓDULO de $2,5 \mathrm{~cm}$. $<>1 "$.

4.- El submódulo sólo tiene carácter aditivo, no pudiéndose usar múltiplos de submódulos.

\section{AUTOMATIZACIÓN DE LA CONSTRUCCIÓN INVISO (Subproyecto 6)}

La industria de la construcción es sin duda una de las áreas económicas y sociales más importantes de nuestro país, aunque su desarrollo tecnológico dista de otros sectores como el del automóvil, el químico o el electrónico. Este hecho encarece el edificio y dificulta determinados aspectos donde la carencia de personal especializado es notable. El elevado coste de los métodos manuales y la ausencia de mano de obra cualificada no son ajenos a la elevada siniestralidad laboral en el sector. El trabajo artesanal en la construcción dificulta también la mejora de otros aspectos característicos en la industrialización como pueden ser la precisión en el trabajo, repetitividad en las operaciones, control sistemático de calidad en los acabados, racionalización y garantía de los suministros, minimización del material almacenado, etc.

La organización y sistematización del proceso constructivo, a semejanza de otras áreas de la producción, ofrece notables ventajas tanto desde el punto de vista del operario, como del inversor o del usuario final del edificio. Ahora bien, las tareas a realizar en la construcción de un edificio son tan numerosas como diversas por lo que su automatización requiere una gran variedad de técnicas y dispositivos para sustituir las diferentes funciones del operario humano. Ello es posible en ciertos casos y puede ser rentable en determinadas condiciones técnico-económicas que condicionarían la difusión del proceso. Así, el enlucido de los muros interiores de una habitación puede realizarse siempre que se desarrolle la estructura móvil para soporte de la boquilla de proyección.

Como alternativa se plantean estrategias de edificación orientadas a la manipulación automatizada en las diferentes fases de ejecución, uso y mantenimiento. El ensamblado de múltiples elementos constructivos modulares y normalizados en sus dimensiones (cubiertas, muros, forjados, ventanas, etc.) contempla incluso espacios tridimensionales de función completa (baños, cocinas, otros). En estos sistemas automáticos el rango de movimiento coincide con las dimensiones del edificio, la carga a manipular ha de superar la del elemento más pesado y la precisión en la manipulación automática la exigida por los sistemas de encastre mecánico entre piezas, típicamente del orden del milímetro.

Con este fin se orienta la búsqueda de un sistema de soporte, movimiento y manipulación de carácter general, capaz de mover cargas elevadas, efectuar trayectorias complejas en un espacio considerable y manipular con precisión objetos de forma, peso y tamaño muy diferente. La imprecisión de los sistemas de carga suspendida y el error acumulado en los brazos hidráulicos articulados han aconsejado la grúa pórtico ligera cuya expansión permite el acceso a planos en cualquier orientación.

Una vez seleccionada, montada y en operación automática la estructura base del robot pórtico se ha realizado para trabajo sobre una vivienda prototipo en el campus del CSIC en Arganda del Rey. Actualmente se ensaya la deposición continua de un material fluido, semi-fluido (mortero, yeso, espumas) o árido, con movimiento del robot sobre un plano vertical y el desarrollo del robot portaherramientas para colocación 
de elementos discretos. Por otro lado, se elaboran los distintos aspectos relacionados con el flujo de información y control durante las operaciones de construcción, uso y mantenimiento del edificio con mayor eficiencia energética, menor coste y mejor seguridad capaces de acceder e interaccionar con las bases de datos de piezas constructivas así como a los planos e instrucciones del diseñador humano.

\section{DESARROLLO DE HERRAMIENTAS INFORMÁTICAS (Subproyecto 7)}

La herramienta informática desarrollada en este subproyecto integrará fundamentalmente los desarrollos logrados en los subproyectos anteriores, sirviendo de interfaz con el proyectista ofreciéndole asistencia técnica y de diseño a la hora de plantear una solución industrializada y sostenible para definir un determinado proyecto constructivo.
La herramienta no pretende ser un sistema "inteligente" de diseño que opere de manera quasi-autónoma inventando "artificialmente" lo que los arquitectos ya han inventado, ya que esto supondría una rigidización del proceso de diseño donde la falta de aportación humana en términos de diseño y adaptación a particularidades relacionadas con el proyecto dificultaría la superación de una de las principales barreras a las que se enfrenta la vivienda industrializada que es la aceptación social.

El subproyecto pretende crear y poner a disposición de los proyectistas un sistema informático "experto" que les ayude en la evaluación y proyecto de diferentes alternativas tanto en el diseño de la vivienda, como en la tipología constructiva a utilizar, buscando aprovechar al máximo las posibilidades que brinda la industrialización, incorporando nuevas tecnologías en materiales y procesos constructivos y que la otorgue al proyectista

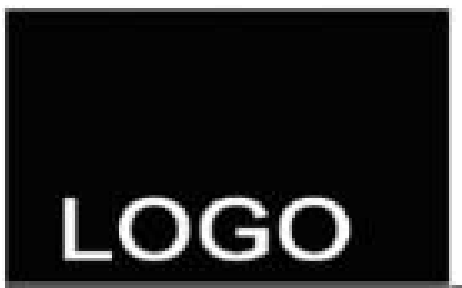

Búsqueda por SISTEMA CONSTRUCTIVO

- Búsqueda por SISTEMA ESPACIAL
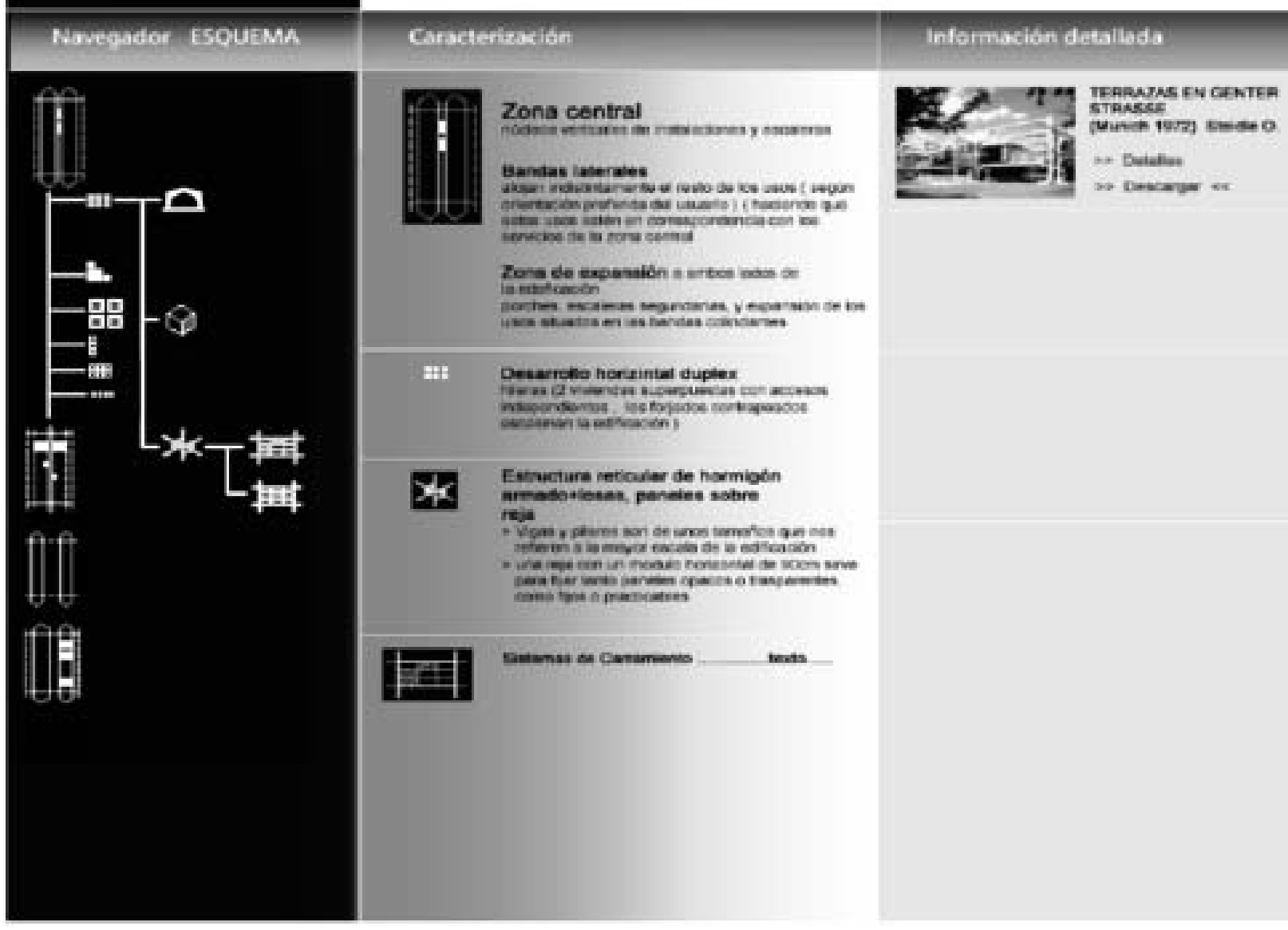


\section{Catálogo de sistemas y componentes constructivos}

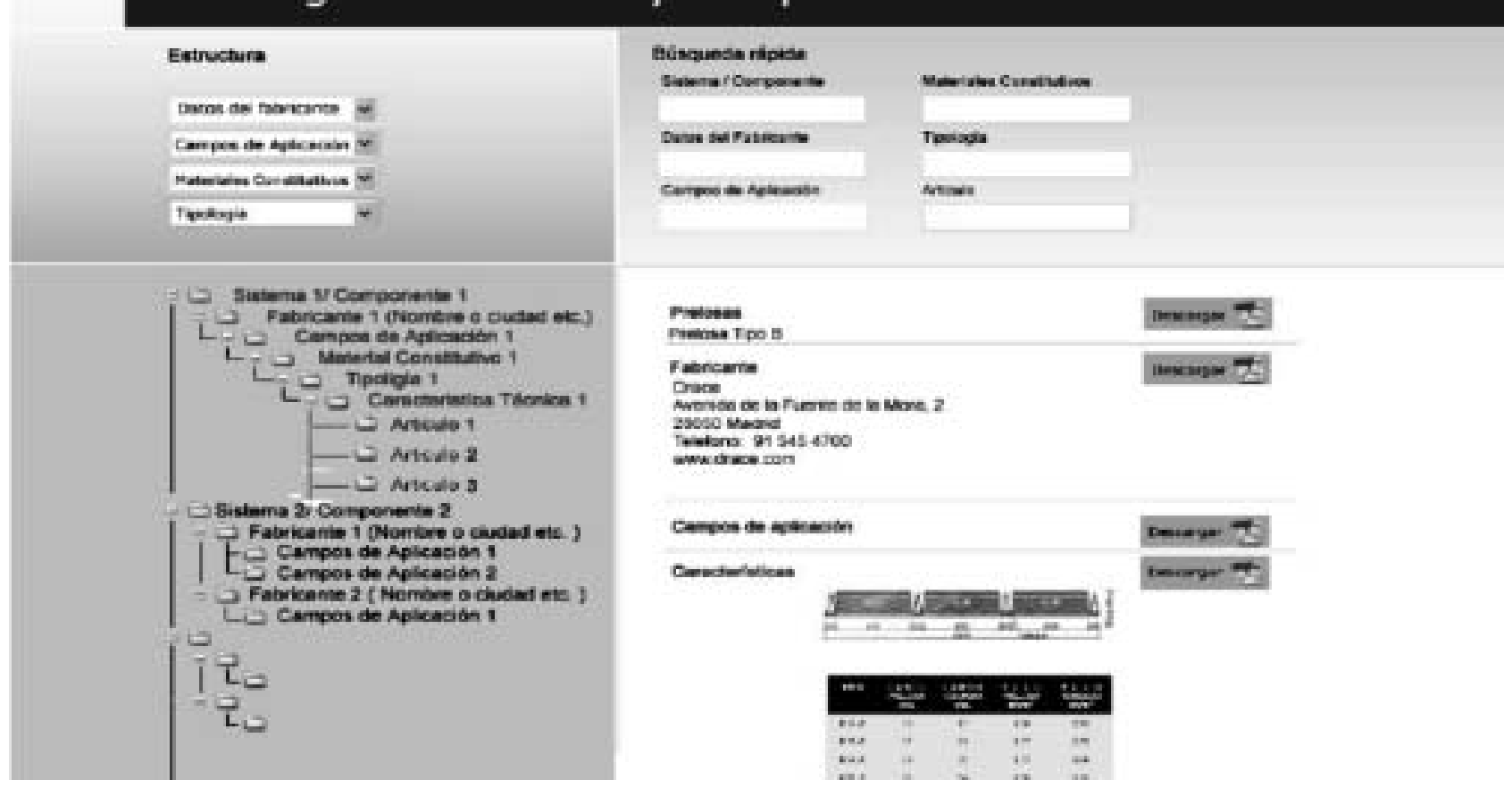

la libertad de operación suficiente para poder adaptar la solución propuesta a los deseos reales de los usuarios a los que va dirigida.

La herramienta presenta dos áreas de desarrollo diferentes que componen una vez integradas el asistente informático completo.

La primera parte que es la que más se ha desarrollado durante el año pasado es el asistente de diseño, el cual engloba una serie de tipologías constructivas desarrolladas en el subproyecto 2, permitiéndonos utilizar cualquiera de ellas en función de las necesidades iniciales del proyecto y posteriormente introducir los cambios deseados.

Dichas tipologías presentarán planos en sistema CAD y todo tipo de información asociada que pueda ser útil al usuario de la herramienta.

La segunda parte del desarrollo incluye un catálogo electrónico para sistemas y componentes constructivos. Hasta ahora se han catalogado dichos componentes y sistemas elaborando una completa base de datos con información sustancial relativa a sus propiedades, características técnicas, dimensiones, etc.... Toda esta información servirá para comenzar el verdadero proceso innovador que ocupa al subproyecto en gran medida durante este año. Este proceso consiste en crear una biblioteca informática de dichos componentes y sistemas, convirtiendo una base de datos en un conjunto de elementos $3 \mathrm{D}$ con sus propiedades integradas de manera que puedan ser incorporadas a las tipologías anteriores, completando desde el punto de vista constructivo la solución propuesta por el proyectista.

La posibilidad brindada a los proyectistas de trabajar con elementos y sistemas constructivos $3 \mathrm{D}$ en vez de con dibujos (líneas) que los simulen, y que además se encuentren agrupados en conjuntos que puedan pertenecer a tipologías constructivas de similares características, les supondrá una gran ayuda en términos de agilización del proceso y de ayuda a tomas de decisión en la definición tanto de diseño como constructiva de soluciones industrializadas para proyectos de construcción.

\section{DISEÑO Y EXPERIMENTACIÓN DE SOLUCIONES TÉCNICAS INNOVADORAS (Subproyecto 9)}

El Subproyecto 9 de Diseño y Experimentación de Soluciones Técnicas Innovadoras del Proyecto INVISO es liderado por el Dr. Arquitecto Sergio Vega Sánchez, en el cual se pretende diseñar nuevas soluciones técnicas y constructivas innovadoras que se puedan aplicar en sistemas modulares de construcción industrializadas que maximicen la calidad y la eficiencia energética de un tipo de vivienda que progresivamente está alcanzando un alto desarrollo en nuestro entorno más competitivo.

El proyecto INVISO profundiza en todas las directrices principales para generar un conocimiento valioso y necesario para la generación de viviendas con un mayor nivel de industrialización y un mayor índice de 
sostenibilidad, analizando en profundidad las distintas variables de la cuestión. El Subproyecto 9, juega en este esquema un papel específico y muy concreto, y es el de profundizar, a partir del análisis general realizado, en el desarrollo de nuevas soluciones técnicas completamente innovadoras para lo que son los sistemas de construcción tradicional, y que puedan aportar conocimiento y experiencias aplicables por la industria en un futuro. Los trabajos de investigación que se tienen previstos para el año 2008, se han articulado en 5 grandes actividades generales y 12 líneas de investigación:

\section{ACTIVIDADES GENERALES A DESARROLLAR SOBRE EL PROTOTIPO EXPERIMENTAL SD7}

\section{A. Construcción de modelos experimentales}

Dentro de esta actividad, se contienen todas las acciones encaminadas a disponer de modelos experimentales sobre los que aplicar los sistemas innovadores diseñados en el Subproyecto, caracterizarlos y optimizarlos.

\section{B. Monitorización de prototipo y modelos experimentales}

Se incluyen todas las acciones encaminadas a la monitorización del prototipo SD7 y de los distintos modelos experimentales que se construyan para caracterizar las variables objeto de análisis.

\section{Instalación de frío y calefacción solar en el prototipo SD7}

En esta actividad están todas las acciones encaminadas a la adaptación del prototipo SD7 para la incorporación de un sistema efectivo de producción de calor y frío solar, mediante una máquina de absorción, y su aplicación a los sistemas de climatización de la casa.

\section{Instalación sistema de energía con pilas} de combustible en prototipo SD7

Se prevé la incorporación de un sistema de energía complementario mediante pilas de combustible, en el cual se aprovecha el excedente de energía eléctrica fotovoltaica para generar hidrógeno y acumularlo en forma de hidruros, de forma que se consiga una mayor autonomía de la casa.

\section{E. Integración de sistemas activos inteligentes y desarrollo de pasarela residencial en prototipo SD7}

Se incluyen todas las acciones orientadas a la integración de todos los sistemas activos

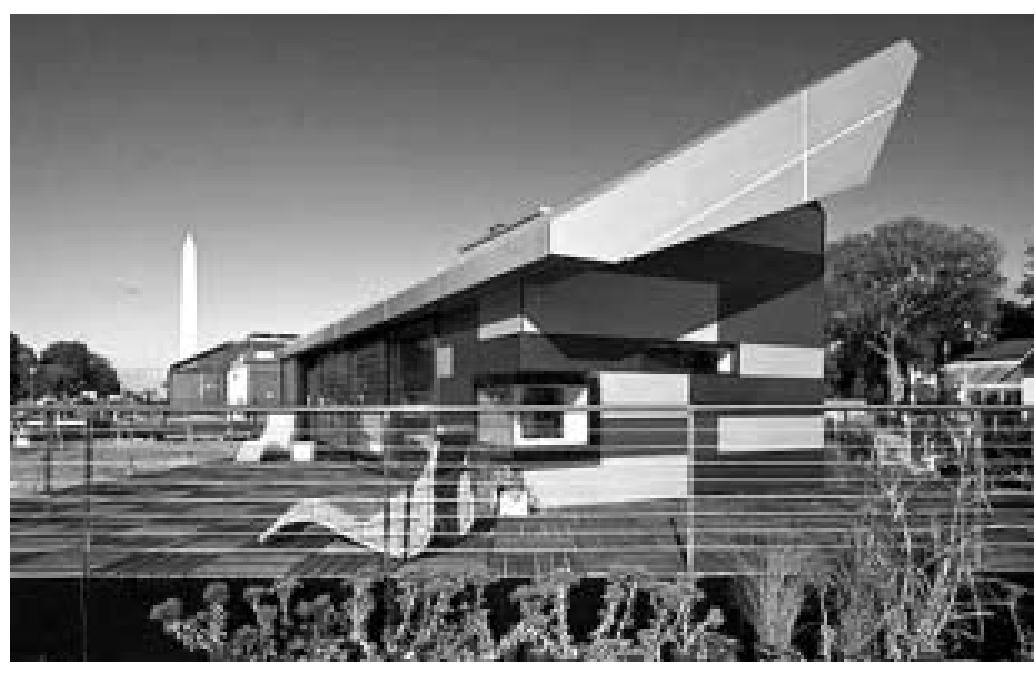

inteligentes de la casa, en la pasarela residen6. Prototipo SD7 cial que debe permitir el funcionamiento del prototipo SD7. Respecto a las líneas de investigación en curso, éstas se pueden articular en cinco categorías, que son:

\section{VERSATILIDAD Y OPTIMIZACIÓN \\ DE SISTEMAS ESTRUCTURALES MODULARES, Y SISTEMAS DE CIMENTACIÓN INTELIGENTE}

\section{I.1. Sistemas de cimentación autonivelables inteligentes}

Se pretende avanzar en el sistema de zapatas que minimicen el impacto real sobre el territorio, y que sean inteligentes, mediante gatos hidráulicos y placas metálicas controladas por presiómetros, rayo laser y un sistema de control de la pasarela residencial.

\section{I.2. Sistemas modulares tridimensionales versátiles}

En esta línea de investigación se pretende desarrollar un sistema estructural y constructivo que permita su empleo como sistemas modulares industrializados tridimensionales, con un fácil transporte de módulos completos tecnológicos que incluyan todos los servicios activos de la vivienda, combinando estos módulos con sistemas 2D.

\section{CARACTERIZACIÓN Y OPTIMIZACIÓN DE CERRAMIENTOS, CUBIERTAS Y SUELOS MULTICAPA LIGEROS DE ALTAS PRESTACIONES}

\section{II.1. Optimización energética de cerramientos multicapa ligeros}

A partir del análisis de las diferentes composiciones de fachadas usadas en las Casas Solares propuestas por las distintas universidades en el Solar Decathlon 2007, se propone una optimización de los cerramientos 


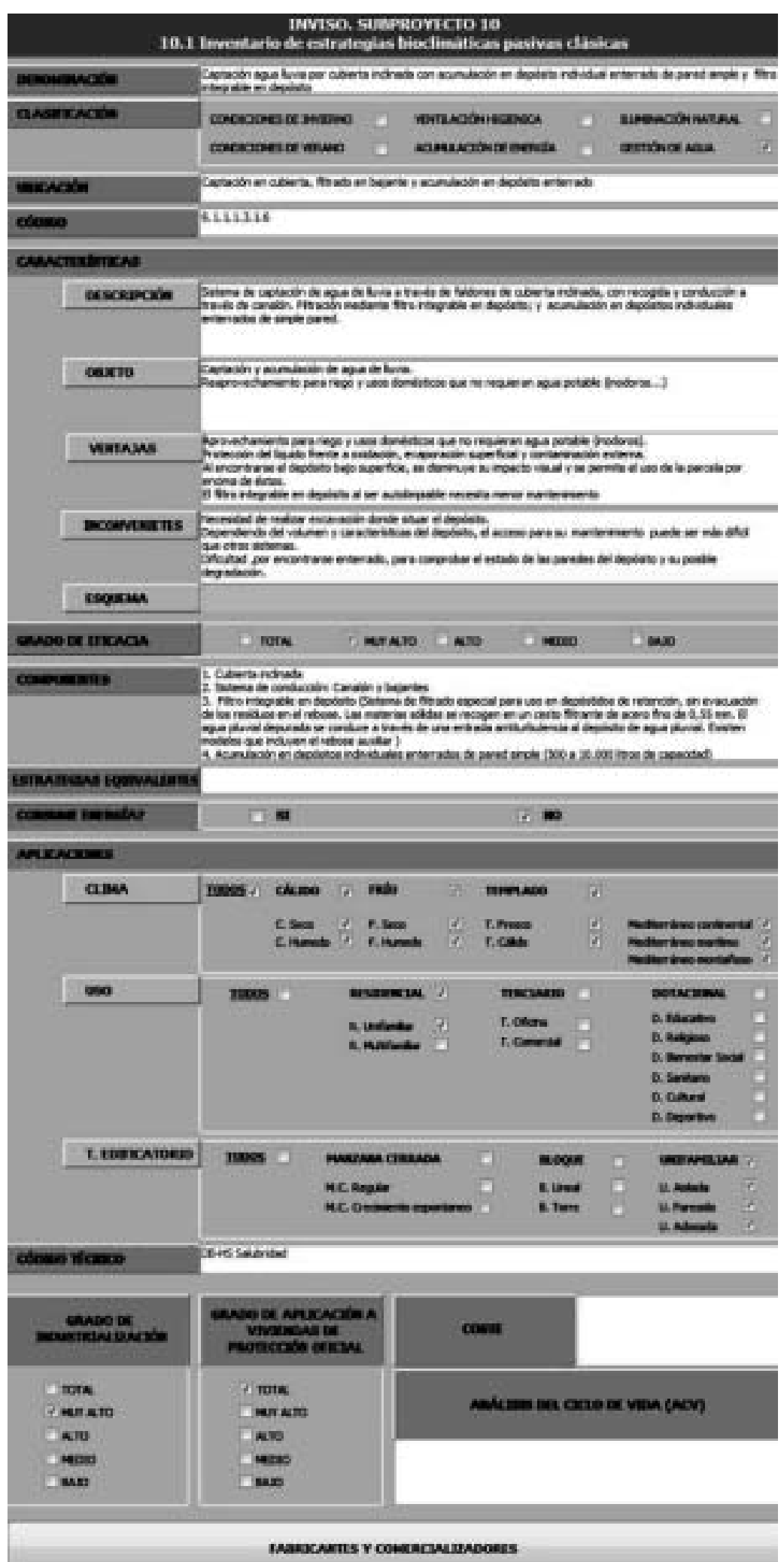

7. Ejemplo de una ficha. multicapa ligeros de altas prestaciones energéticas.

II.2. Optimización del coste de ciclo de vida de cerramientos multicapa ligeros

Optimización de los cerramientos multicapa ligeros diseñados y propuesta de nuevos materiales para mejorar el comportamiento desde el punto de vista de impacto ambiental y factor biótico.

\section{II.3. Optimización de cerramientos. Integración de sistemas solares activos en cerramientos de viviendas}

Generar conocimiento para promover la integración de los sistemas solares activos en cerramientos de viviendas, profundizando especialmente en las condiciones para una adecuada integración arquitectónica y tecnológica, y con especial estudio en sistemas híbridos.

\section{II.4. Optimización acústica de cerramientos multicapa ligeros}

Se caracterizarán y optimizarán las distintas soluciones multicapas ligeras desde el punto de vista de aislamiento acústico, tanto frente a impacto, como frente a ruido aéreo, de forma que se puedan conseguir cerramientos ligeros de altas prestaciones energéticas, sin menoscabo de sus condiciones de confort acústico.

\section{OPTIMIZACIÓN DE SISTEMAS DE DOBLE PIEL Y VENTANA INTELIGENTE}

\section{III.1. OPTIMIZACIÓN DE DOBLES PIELES INTELIGENTES}

Generar conocimiento para posibilitar el empleo de grandes cerramientos de vidrio, de modo que se minimice el impacto negativo sobre el comportamiento energético del edificio, recurriendo a técnicas de apantallamiento selectivo, y de control de aireadores que aprovechen las posibilidades de las cámaras exteriores de la doble piel.

\section{III.2. OPTIMIZACIÓN DE VENTANAS Y VENTANAS INTELIGENTES}

Se persigue el generar el conocimiento necesario para la optimización energética, lumínica, acústica y económica de las ventanas recurriendo a sistemas pasivos como orientaciones, formatos, proporciones, apantallamientos,... y a sistemas activos mediante aireadores, pantallas selectivas, tipos de vidrio, etc., gobernados por la pasarela residencial de la casa.

\section{OPTIMIZACIÓN DE SISTEMAS DE DIVISIÓN INTERIOR E INSTALACIONES}

IV.1. Optimización del sistema de tabiques, forjados, y divisiones interiores

Explorar todas las posibilidades que presentan los sistemas de construcción ligeros para las tabiquerías, forjados, y demás elementos interiores, para la generación de cámaras in- 
termedias que optimicen las prestaciones de los elementos en el comportamiento eficiente de las viviendas.

\section{IV.2. Optimización de sistemas de integración e interconexión de instalaciones}

Ver todas las posibilidades que presentan los sistemas de construcción ligeros para facilitar el tendido de instalaciones de forma que sean funcionales, versátiles, accesibles, seguras, y que permitan un eficaz y sencillo sistema de conexión y desconexión, así como condiciones de accesibilidad y registro.

\section{UTILIZACIÓN DE SISTEMAS ACTIVOS Y PASIVOS EN SOLUCIONES MULTICAPA.}

\section{V.1. Sistemas de acumulación de frío y calor solar, y aplicación de sistemas de precalentamiento y pre-enfriamiento del aire de ventilación de las viviendas}

Se busca una forma eficiente de acumulación del calor y frío solar generado por paneles térmicos y máquinas de absorción, mediante elementos de gran inercia térmica como son los CPM (geles de cambio de fase), y de forma que, además de las obvias aplicaciones para la climatización, sirva para el pretratamiento del aire de ventilación de la vivienda, a un coste energético despreciable.

\section{V.2. Integración de sistemas de recuperación de calor de sistemas de ventilación, en las soluciones multicapa ligeras}

Integración de los sistemas de recuperación de calor residual de las viviendas, e incorporarlos en los multicapas, de forma que el aire de ventilación de las mismas, antes de su expulsión, extraigamos el calor o el frío residual para pre-acondicionar el aire de ventilación entrante.

En este Subproyecto participan varias OPIs como la Universidad Politécnica de Madrid (UPM-TISE): y el Instituto de Automática Industrial (IAI) e Instituto de Ciencias de la Construcción Eduardo Torroja (IETcC) pertenecientes al Consejo Superior de Investigaciones Científicas. Igualmente participan empresas privadas como Soluciones de Edificación Integrales y de Servicios-SEIS, heredera de DRACE. DRAGADOS. BLUECOAST. CIDEMCO. GOP Y VEKA.

\section{SISTEMAS PARA LA OPTIMIZACIÓN DEL COMPORTAMIENTO EFICIENTE DE LAS VIVIENDAS (Subproyecto 10)}

El subproyecto 10 lo lidera el grupo de investigación reconocido por la Universidad
Politécnica de Madrid ABIO, Arquitectura Bioclimática en un Entorno Sostenible, cuyas líneas de investigación son los materiales sostenibles y las estrategias y actuaciones arquitectónicas y urbanísticas bioclimáticas.

La investigación fundamental desarrollada durante este año fue la catalogación de estrategias bioclimáticas aplicables a la arquitectura, organizadas en forma de árbol. Posteriormente, con un criterio de selección científico basado en indicadores, se han elegido, para su desarrollo constructivo, aquellas que pudieran ser industrializables y caber dentro de los objetivos de la sostenibilidad aplicada a las viviendas de protección oficial.

Se ha estructurado en seis macro-grupos: 1. Estrategias utilizadas en condiciones de invierno; 2. Estrategias utilizadas en condiciones de verano; 3. Ventilación higiénica; 4. Acumulación de energía; 5. Iluminación natural, y 6. Gestión del agua.

Hasta el momento el árbol se compone de 841 entradas; cada una de ellas representa una estrategia diferente. La caracterización detallada de las estrategias se ha sistematizado a través de fichas. Las fichas se componen de varios apartados, con el objetivo de describir detalladamente la estrategia: Denominación, Clasificación, Ubicación en el edificio, Código Características (incluyendo un enlace a un fichero donde está dibujada la estrategia), Grado de eficacia, Componentes, Estrategias equivalentes, Consumo de energía, Aplicaciones (según el clima, el uso y la tipología), Código Técnico, Grado de industrialización, Grado de aplicación a viviendas de protección oficial, Coste y Análisis del ciclo de vida.

Un enlace denominado fabricantes y comercializadores permite abrir otra ficha donde se enumeran las empresas que fabrican o comercializan productos asimilables a la estrategia descrita.

Se ha iniciado ya el desarrollo de algunas de las estrategias previamente elegidas de entre las fichas. Estas estrategias serán líneas prioritarias para los trabajos de años posteriores. Una de esa líneas es el Desarrollo y análisis de los sistemas de acumulación de calor en sustancias de cambio de fase. A ella corresponden varias ramas del árbol.

La primera consiste en el empleo de Materiales de Cambio de Fase (PCM), en forma de parafinas microencapsuladas mezclada con yeso para fabricar paneles de yeso laminado (PYL). Una segunda rama comprende las fichas que desarrollan el empleo de PCM en forma de parafinas macroencapsuladas situ- 


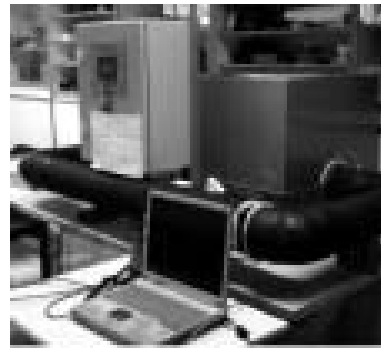

adas en un suelo técnico ventilado. La tercera rama es el empleo de PCM de parafinas a granel dentro de un contenedor en forma de baldosa de pavimento. En la actualidad se está ensayando con la baldosa colocada sobre suelo técnico, pero se va a iniciar el ensayo con las baldosas directamente sobre el forjado.

Otra de las líneas prioritarias es el Desarrollo y análisis de las envolventes vegetales. Un primer apartado de esta investigación consiste en el desarrollo de un sistema industrializable de cubiertas vegetales extensivas aljibe prevegetadas.
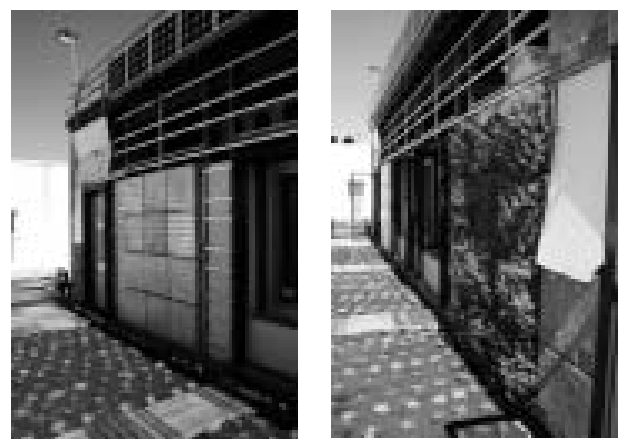

de determinar el ahorro energético y los beneficios ambientales de las fachadas vegetales en comparación con las fachadas convencionales, se ha puesto en marcha un proyecto de monitorización de dos diferentes tipos de fachada en un edificio. Los dos tipos de fachadas que se están montando son una fachada vegetal (con paneles removibles prevegetados y autorregables) y una fachada traslucida (elemento de vegetación fijo, deslizable o permeable sobre una estructura semitrasparente). Este segundo módulo que se está montando tiene el objetivo de desarrollar un sistema de fachada translúcida que incorpore vegetación, con el objeto de
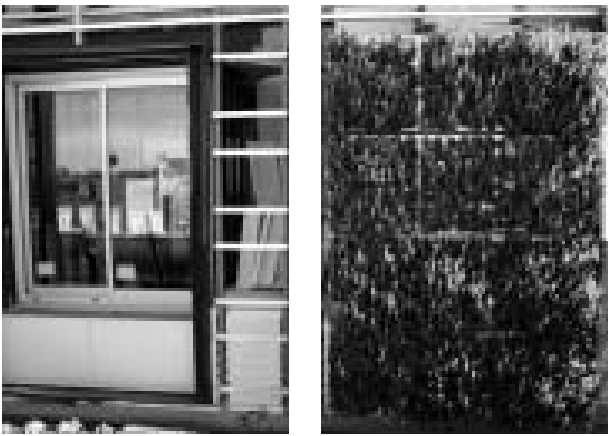
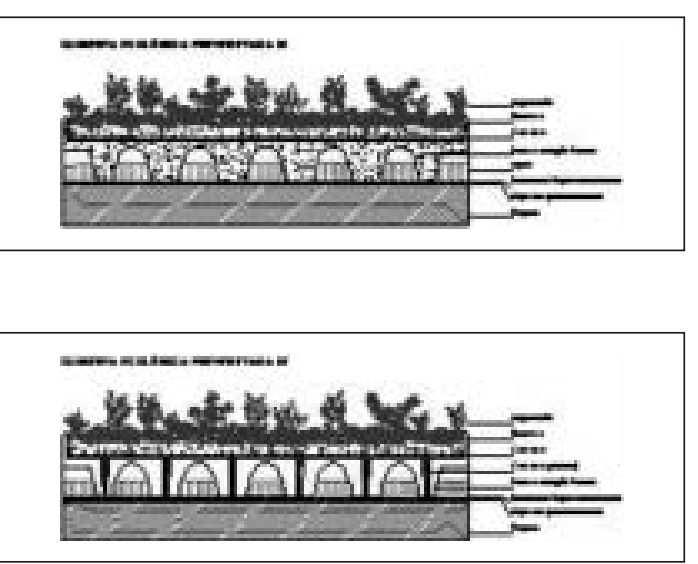
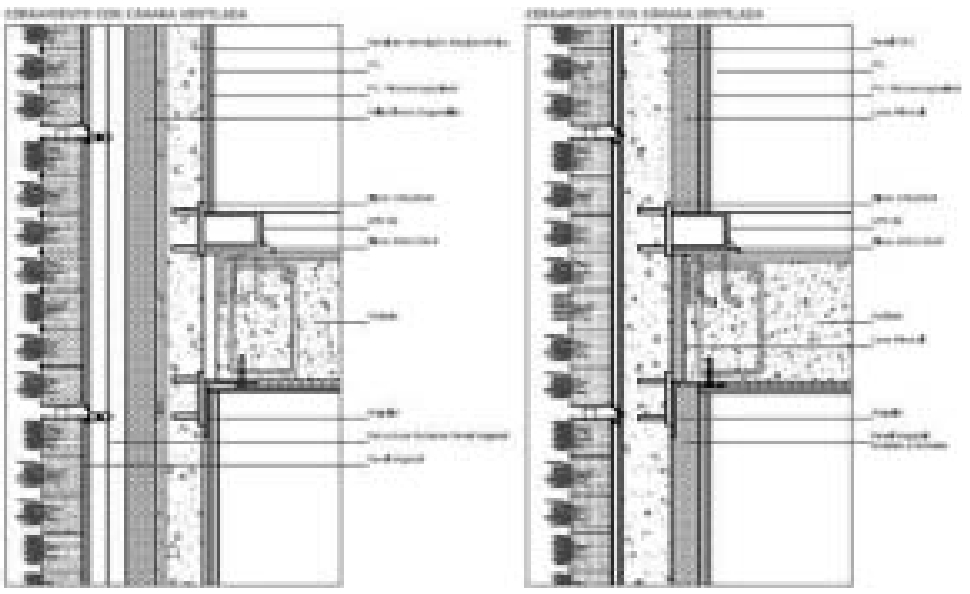

8. Equipo de medida de la capacidad acumuladora de los PYL con PCM empleado.

9. Demostrador donde se han montado los macroencapsulados de PCM.

10. Macroencapsulados de PCM empleados en este ensayo.

11. La fachada vegetal en su fase de montaje. Se puede ver la fachada con paneles prevegetados montada y la fachada testigo sin vegetación.

12. Panel prevegetado para cubierta aljibe y fachada vegetal prevegetada.

13. Módulos de la fachada translúcida en un momento de su construcción.
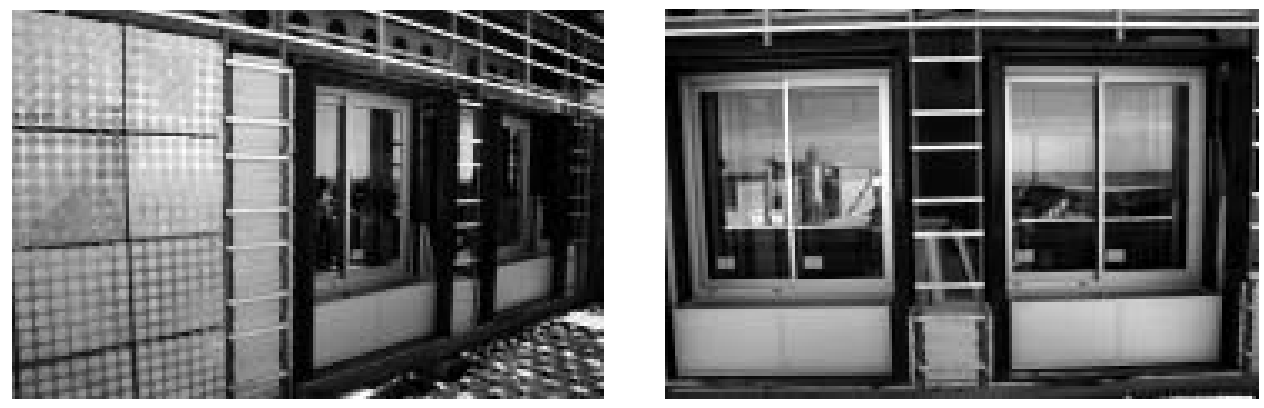

El segundo de los apartados de esta línea estratégica es la del desarrollo de una fachada vegetal. Con el objetivo de demostrar y analizar su influencia en el control lumínico, acústico e higrométrico de los ambientes interiores. 